\title{
Coupled Fluid-Structure Transient Thermal Analysis of a Gas Turbine Internal Air System with Multiple Cavities
}

\author{
Vlad Ganine; Umesh Javiya, Nick Hills, John Chew \\ Thermo-Fluid Systems UTC \\ Faculty of Engineering and Physical Sciences \\ University of Surrey \\ Guildford, Surrey GU2 7XH, UK
}

\begin{abstract}
This paper presents the transient aero-thermal analysis of a gas turbine internal air system through an engine flight cycle featuring multiple fluid cavities that surround a HP turbine disk and the adjacent structures. Strongly coupled fluid-structure thermal interaction problems require significant computational effort to resolve nonlinearities on the interface for each time step. Simulation times may grow impractical if multiple fluid domains are included in the analysis. A new strategy is employed to decrease the cost of coupled aero-thermal analysis. Significantly lower fluid domain solver invocation counts are demonstrated as opposed to the traditional coupling approach formulated on the estimates of heat transfer coefficient. Numerical results are presented using $2 D F E$ conduction model combined with $2 D$ flow calculation in five separate cavities interconnected through the inlet and outlet boundaries. The coupled solutions are discussed and validated against a nominal stand-alone model. Relative performance of both coupling techniques is evaluated.
\end{abstract}

\footnotetext{
Nomenclature

$\mathcal{F}$ fluid solver

$I$ identity matrix

K conductivity matrix

M capacitance matrix

Q heat flux

$\mathcal{S}$ solid solver

T temperature

$h$ heat transfer coefficient

n normal component

$\mathbf{r}$ temperature residual

$t$ time
}

\section{Greek}

$\beta$ relaxation coefficient 
$\gamma$ least-squares problem solution

$\Delta$ difference

$\delta$ wall temperature perturbation factor

$\theta$ time discretization control parameter

\title{
Superscripts
}

()$^{n} \quad$ n-th time step

\section{Subscripts}

()$_{N} \quad \mathrm{~N}$-th fluid domain

()$_{f}$ defined on fluid domain

()$_{k} \quad \mathrm{k}$-th iteration

()$_{s}$ defined on solid domain

()$_{\Sigma}$ defined on interface $\Sigma$

\|\|$_{2} \quad l^{2}$ norm

\author{
Acronyms \\ $C F D$ computational fluid dynamics \\ $F E$ finite element \\ $H P$ high pressure \\ $I P$ intermediate pressure \\ $L P$ low pressure
}

\section{INTRODUCTION}

Accurate prediction of aerodynamic, aero-mechanical and thermo-mechanical phenomena attracts an increasing interest from the gas turbine industry. Efficient and robust analysis procedures able to properly describe the thermal and flow environment within a secondary air system may lead to substantial gains in overall engine performance, weight and components reliability by offering improved means of optimizing designs [1].

Typically, in thermal modeling, the internal air system is modeled with user-specified boundary conditions, while more accurate CFD predictions, if available, are applied only on some portions of the system. The user-specified boundary conditions rely heavily on correlations, they require a significant effort from an end-user to correctly represent the complex physical phenomena over a wide range of operating conditions. As the geometries of modern air systems grow in complexity, current models with correlations become painful to build and increasingly obsolete.

A general trend in computational modeling of modern turbo-machinery systems is to move towards the "virtual" or "whole" engine simulation [2]. As far as modeling of the internal air systems is concerned, a natural and incremental advance in both the complexity and the accuracy of current modeling is to include and interconnect multiple CFD domains within a single simulation. Among the advantages it may offer are a lower level of human intervention and time required to set up the models, and more importantly, automatic generation of the boundary conditions for the downstream components. However, this comes at a price of a considerably higher computational effort required to run a simulation through an engine transient flight cycle leading to long analysis times.

Many studies in recent years sought to improve the predictive capabilities of thermo-mechanical analysis codes by coupling FE solvers to detailed CFD models of individual components to more accurately evaluate wall temperature distribution in turbine cavities, see, for example, $[3,4,5,6,7]$. While these studies were able to obtain only a general agreement with the experimental data, they did demonstrate many of the fundamental features outlined in earlier investigations. Subsequent efforts attempted to further improve the agreement by including some of both fluid and solid domains 3D geometrical features in the analysis [8] or the effects of solid domain thermo-mechanical distortion on flow dynamics [9]. Still, accurate and automatic predictions of heat transfer in the internal air systems remain a difficult challenge.

The main goal of this paper is to provide a snapshot of the state-of-the-art FE/CFD coupled analysis capability using an industrial scale model of an operating engine that features multiple cavities interconnected through the inlet-outlet boundaries. Full transient analysis is conducted through an engine flight cycle to obtain HP (high pressure) turbine disk 
temperature profiles, which are compared to the baseline stand-alone model predictions. A new more advanced coupling technique, inspired by the work of Anderson [10], is employed to accelerate the analysis. It is shown that the Anderson mixing method offers a considerable, up to $\mathrm{x} 2$, reduction of total analysis time compared to the currently in use method based on the convective heat flux formulation.

The rest of the paper is outlined as follows: in Sec. 2, we review the strong coupling partitioned methodology for conjugate heat transfer analysis. Both, the convective method and the Anderson mixing techniques are formulated; and in Sec. 3, we present the test case experimental setup. Some wall temperature distribution results of the coupled simulation are given in Sec. 4, where the relative computational performance of two coupling techniques is compared on the selected test case as well. Concluding remarks are given in Sec. 5.

\section{NUMERICAL APPROACH}

\section{Coupling Strategies}

Conjugate heat transfer problems belong to the surface coupled class of multi-physics systems that can be solved either with monolithic or partitioned approaches. Monolithic methods incorporate different physics phenomena over multiple domains in a single highly specialized solver (typically a modified CFD code, see, for example, $[11,5,12]$ ), but generally they lack modeling flexibility. More flexible and reliable partitioned approaches solve the fluid and solid problems separately with the existing codes subject to complementary coupling transmission conditions. Stability analysis of the partitioned schemes for thermally coupled fluid-structure problems is presented in $[13,14]$. These results suggest that for stability reasons the best choice is a classical partitioning scheme known as Dirichlet-Neumann, where the the fluid problem is supplemented with the Dirichlet boundary condition (temperature), and the Neumann condition (heat flux) is imposed on the solid domain. Thus at the interface between fluid and solid regions the matching conditions to satisfy are the continuity of the temperature and the normal component of heat flux

$$
\begin{aligned}
\mathbf{T}_{\Sigma, f} & =\mathbf{T}_{\Sigma, s} \\
\mathbf{n}_{f} \cdot \mathbf{Q}_{\Sigma, f} & =-\mathbf{n}_{s} \cdot \mathbf{Q}_{\Sigma, s}
\end{aligned}
$$

Weakly coupled strategies do not enforce the continuity exactly at each time step. Many practical coupled heat transfer problems can be particularly difficult to treat explicitly for a certain ratio of characteristic time scales between adjacent solid and fluid regions due to inherent numerical instabilities. In these situations strongly coupled or implicit partitioned approaches are unavoidable. They require a higher frequency of exchanges between the solvers at each time step to meet a convergence criterion of the following nonlinear system of equations defined on the interface

$$
\left\{\begin{array}{l}
\mathbf{Q}_{\Sigma}^{n+1}=\mathcal{F}\left(\mathbf{T}_{\Sigma}^{n+1}\right) \\
\mathbf{T}_{\Sigma}^{n+1}=\mathcal{S}\left(\mathbf{Q}_{\Sigma}^{n+1}\right)
\end{array}\right.
$$

The system can be equivalently viewed as a fixed point problem

$$
\mathbf{T}_{\Sigma, k+1}^{n+1}=\mathcal{S}\left(\mathcal{F}\left(\mathbf{T}_{\Sigma, k}^{n+1}\right)\right)
$$

The major concern with direct application of the fixed-point iteration procedure is that it may not converge at all, or at the best, exhibit only linear convergence rates. Many realistic thermal coupled problems are particularly prone to oscillations that ultimately lead to a divergent iterative process. A number of techniques have been proposed that increase stability of the iterative scheme, and in some cases accelerate the linear convergence as well. In this work we compare a numerical scheme based on convective formulation of the heat flux with estimation of the heat transfer coefficients to the Anderson acceleration procedure. Both techniques are presented in the following subsections. 


\section{Convective Formulation of Heat Flux}

According to the Newton's law of cooling the normal component of heat flux can be expressed as

$$
Q_{\Sigma}^{n+1}=h\left(T_{\Sigma, s}^{n+1}-T_{\Sigma, f}^{n+1}\right)
$$

This equation provides an implicit relation between the wall temperature $T_{\Sigma, s}^{n+1}$ transmitted to the fluid solver and the heat flux solution $Q_{\Sigma}^{n+1}$ imposed on the solid domain boundary. The major issue is how to evaluate the heat transfer coefficients $h$. A simpler approach pursued in $[3,15,16]$ is to select a constant positive value of $h$. In that case the scheme may be viewed as a form of under-relaxation of each iteration that increases the stability yet does not improve the linear convergence rate of the fixed-point iteration process.

An alternative approach is proposed in [4] and applied to coupled thermal analysis of an internal air system in [7,8]. It is based on the observation that the effect of changes in wall temperature $T_{\Sigma, s}^{n+1}$ on the heat transfer coefficient $h$ due to fluid property variations is rather negligible. Therefore a valid estimate of the heat transfer coefficient can be obtained with an extra call to the fluid solver subject to a slightly perturbed wall temperature condition $T_{\Sigma, \delta}^{n+1}=T_{\Sigma, s}^{n+1}+\delta$ as

$$
h=\frac{Q_{\Sigma}^{n+1}-Q_{\Sigma, \delta}^{n+1}}{T_{\Sigma, s}^{n+1}-T_{\Sigma, \delta}^{n+1}}
$$

Note that $h$ here is nothing more than a finite difference approximation of the derivative $\partial Q_{\Sigma}^{n+1} / \partial T_{\Sigma}^{n+1}$. After integration along the interface $h$ contributes to the surface conductivity matrix in the nodal space of the solid solver. The iterative procedure to retrieve unknown wall temperature is illustrated as follows. Assuming FE discretization in space and the generalized trapezoidal difference rule for discretization in time the solid domain nonlinear residual is given by

$$
\mathbf{r}=\frac{\mathbf{M}}{\Delta t}\left(\mathbf{T}^{n}-\mathbf{T}^{n+1}\right)-\mathbf{K}\left((1-\theta) \mathbf{T}^{n}+\theta \mathbf{T}^{n+1}\right)+\mathbf{Q}^{n+1}\left(\mathbf{T}^{n+1}\right)
$$

The unknown vector of nodal temperatures $\mathbf{T}^{n+1}$ is iterated until convergence using the quasi-Newton update formula given by

$$
\mathbf{T}_{k}^{n+1}=\mathbf{T}_{k-1}^{n+1}-\widehat{\mathbf{J}}^{n+1^{-1}} \mathbf{r}_{k}^{n+1}
$$

where the approximate Jacobian $\partial \mathbf{r} / \partial \mathbf{T}^{n+1}$ is evaluated once at the beginning of a time step $t^{n+1}$ as

$$
\widehat{\mathbf{J}}^{n+1}=\frac{\partial \mathbf{Q}}{\partial \mathbf{T}^{n+1}}-\frac{\mathbf{M}}{\Delta t}-\theta \mathbf{K}
$$

with $\partial \mathbf{Q} / \partial \mathbf{T}^{n+1}$ replaced by the surface conductivity matrix. Note that unlike in many strongly coupled partitioned approaches defined on the interface, this one treats the fluid domain state as an internal variable of the solid problem.

\section{Anderson Acceleration}

Instead of estimating the heat transfer coefficient at the cost of an extra fluid solver call, a higher quality and more recent information on $\partial Q_{\Sigma}^{n+1} / \partial T_{\Sigma}^{n+1}$ can be obtained directly from the nonlinear iteration sequence by applying the Anderson mixing procedure [10]. Detailed performance analysis of the method with application to the conjugate heat transfer problems is given in [17]. The procedure starts with an initial guess of the interface temperature field $\mathbf{T}_{\Sigma, 0}^{n+1}$ applied as a boundary 
condition to the fluid solver. The latter provides a heat flux solution fed back to the solid solver to obtain a new temperature estimate $\tilde{\mathbf{T}}_{\Sigma, 0}^{n+1}=\mathcal{S}\left(\mathcal{F}\left(\mathbf{T}_{\Sigma, 0}^{n+1}\right)\right)$. The interface residual is formed as

$$
\mathbf{r}_{\Sigma, 0}^{n+1}=\tilde{\mathbf{T}}_{\Sigma, 0}^{n+1}-\mathbf{T}_{\Sigma, 0}^{n+1}
$$

Before the Anderson process can start, a second residual vector $\mathbf{r}_{\Sigma, 1}^{n+1}$ has to be evaluated with a call to fluid solver using a simple under-relaxation technique with coefficient $0<\beta<1$

$$
\mathbf{T}_{\Sigma, 1}^{n+1}=\mathbf{T}_{\Sigma, 0}^{n+1}+\beta \mathbf{r}_{\Sigma, 0}^{n+1}
$$

Two vector differences are formed that essentially contain the most up-to-date information on our approximated Jacobian

$$
\begin{aligned}
& \Delta \mathbf{T}_{\Sigma, 1}^{n+1}=\mathbf{T}_{\Sigma, 0}^{n+1}-\mathbf{T}_{\Sigma, 1}^{n+1} \\
& \Delta \mathbf{r}_{\Sigma, 1}^{n+1}=\mathbf{r}_{\Sigma, 0}^{n+1}-\mathbf{r}_{\Sigma, 1}^{n+1}
\end{aligned}
$$

Second and subsequent iterations are carried out with the Anderson update formula as

$$
\mathbf{T}_{\Sigma, k+1}^{n+1}=\mathbf{T}_{\Sigma, k}^{n+1}+\beta \mathbf{r}_{\Sigma, k}^{n+1}+(\Delta \mathbf{T}+\beta \Delta \mathbf{R}) \boldsymbol{\gamma}
$$

where the differences from current and all previous iterations are accumulated as the columns of matrices $\Delta \mathbf{T}$ and $\Delta \mathbf{R}$

$$
\begin{aligned}
& \Delta \mathbf{T}=\left[\Delta \mathbf{T}_{\Sigma, 1}^{n+1}, \ldots, \Delta \mathbf{T}_{\Sigma, k}^{n+1}\right] \\
& \Delta \mathbf{R}=\left[\Delta \mathbf{r}_{\Sigma, 1}^{n+1}, \ldots, \Delta \mathbf{r}_{\Sigma, k}^{n+1}\right]
\end{aligned}
$$

and the vector $\boldsymbol{\gamma}$ is obtained by solving an unconstrained least-squares problem

$$
\min _{\left[\gamma_{1}, \ldots, \gamma_{k}\right]}\left\|\mathbf{r}_{\Sigma, k}^{n+1}+\sum_{i=1}^{k} \gamma_{i} \Delta \mathbf{r}_{\Sigma, i}^{n+1}\right\|_{2}
$$

Whenever the residual falls below a certain suitably small tolerance level, the interface temperature solution for the current time step is accepted. Note that unlike in the previous method before the wall temperature is passed to a CFD solver the solid sub-problem is iterated to a full convergence. The coupling algorithm for one time step is illustrated in Alg. 1.

To further reduce the number of iterations per time step, one can incorporate data accumulated at $j$ previous time steps as

$$
\begin{aligned}
\Delta \mathbf{T} & =\left[\Delta \mathbf{T}_{\Sigma, 1}^{n+1-j}, \ldots, \Delta \mathbf{T}_{\Sigma, k}^{n+1}\right] \\
\Delta \mathbf{R} & =\left[\Delta \mathbf{r}_{\Sigma, 1}^{n+1-j}, \ldots, \Delta \mathbf{r}_{\Sigma, k}^{n+1}\right]
\end{aligned}
$$

The number of time steps to reuse that results in the optimal performance is rather problem-specific. In this case study we employ the reuse of data from one previous time step. 


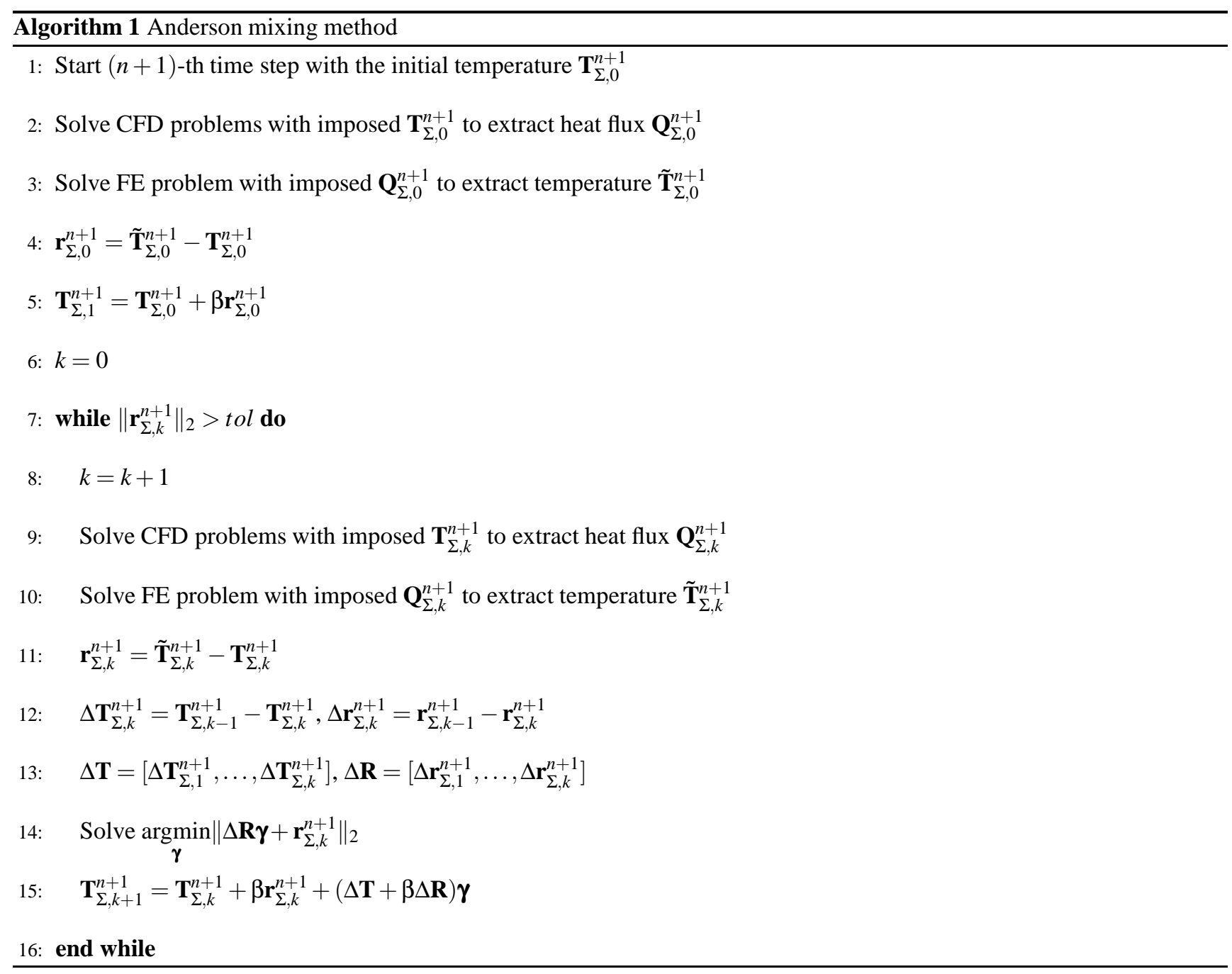

\section{Multiple Cavities Implementation}

A single mixing parameter $\beta$ may not suffice to ensure optimum performance when multiple fluid domains are included. Large oscillations may arise in some fluid cavities that feature slender parts of the metal with a certain ratio of thermal conductivity and diffusivity between the adjacent fluid and solid areas. By suitably choosing a smaller parameter $\beta$ to ensure convergence in these cavities, problems may arise with the iteration process converging very slowly in the others. Thus, the optimum value of $\beta$ for each domain should be adjusted empirically, which leads to the following update formula

$$
\mathbf{T}_{\Sigma, k+1}^{n+1}=\mathbf{T}_{\Sigma, k}^{n+1}+\mathbf{B}_{0} \mathbf{r}_{\Sigma, k}^{n+1}+\left(\Delta \mathbf{T}+\mathbf{B}_{0} \Delta \mathbf{R}\right) \boldsymbol{\gamma}
$$

where

$$
\mathbf{B}_{0}=\left(\begin{array}{ccc}
\beta_{1} I & \ldots & 0 \\
\vdots & \ddots & \vdots \\
0 & \ldots & \beta_{N} I
\end{array}\right)
$$

Since the outlet temperature of upstream CFD models may be connected to the inlets of the downstream cavities through the standard convective correlations, variable strength of nonlinear coupling exists between fluid domains through the inlet- 
outlet boundaries. To ensure the optimal convergence for strongly coupled cases, the vector of interface unknowns $\mathbf{T}_{\Sigma}^{n+1}$ can be extended to also include the inlet temperatures.

\section{TEST CASE DESCRIPTION}

\section{Solid Domain}

The test case selected for this work is a HP turbine disk surrounded by five cavities interconnected through the labyrinth seals. Since we employ transient thermal analysis only, the solid domain boundaries in FE model remain stationary. An axisymmetric FE model of HP turbine disc with surrounding parts is given in Fig. 1. The model is composed of a HP

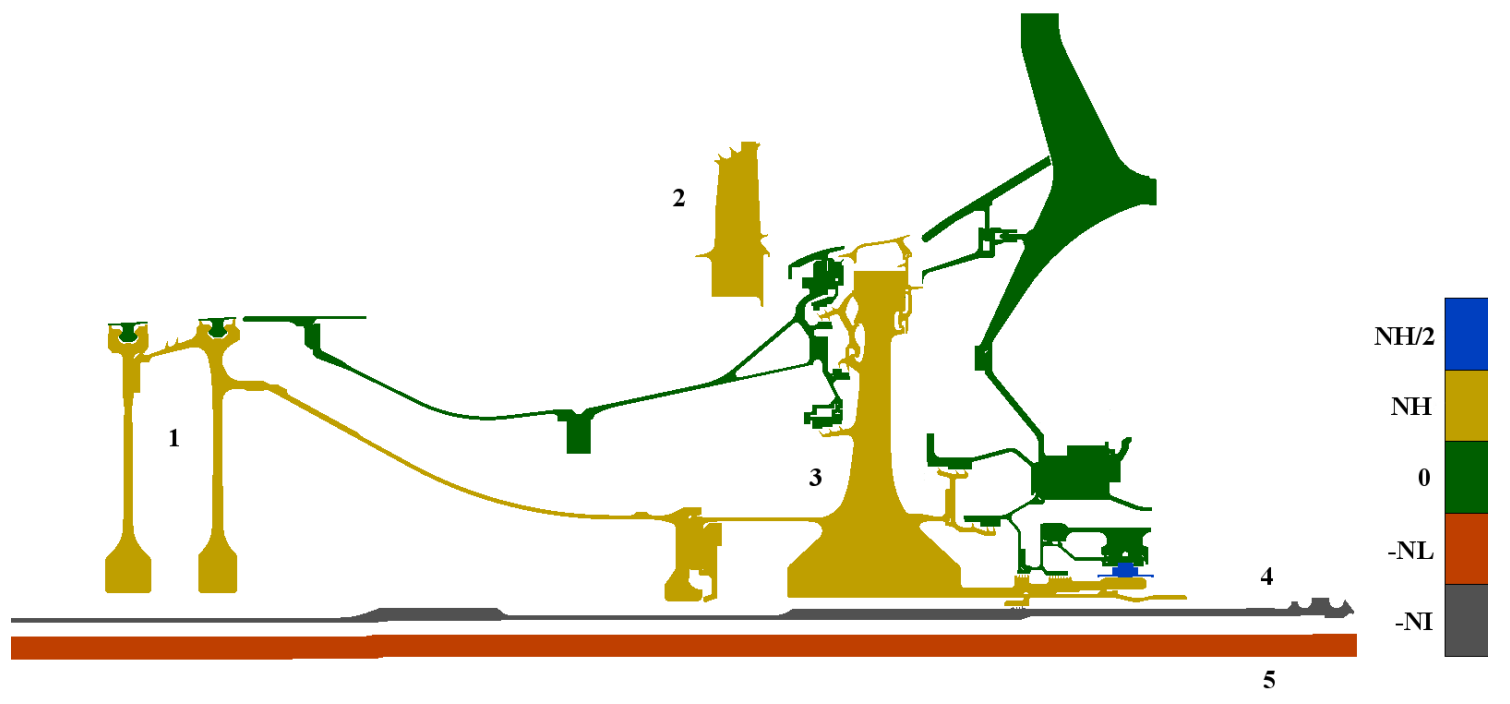

Fig. 1. Axisymmetric model of HP rotor assembly showing each component's rotational speed.

turbine disk and blade marked (3) and (2) correspondingly, two HP compressor stages (1), an IP shaft (4) and an LP shaft (5). All of these parts are surrounded by stationary components. Non-axisymmetric features such as holes and the blade are modeled using an equivalent axisymmetric thicknesses of the metal. The HP blade is moved from its original position both to clarify the model and to handle its complex hollow geometry with cooling passages and holes. Nonetheless, the blade is kinematically and thermally linked to the main model through continuous boundaries. Solid metal components are colored according to their angular velocities with NH, NI and NL denoting high, intermediate and low pressure turbine speeds accordingly. The partition of the solid domain is extracted from a full FE model of a turbine engine to reduce calculation time, while maintaining the same level of accuracy. This results in a FE model featuring 13,383 six node quadratic triangular elements with a total of 31,305 nodes.

In the stand-alone baseline FE model, the heat transfer boundaries of the cavity walls are represented by the standard heat transfer correlations. The latter are tuned to match the engine test measurements. The stand-alone FE model "model matching" procedure is described by Dixon et al. in [18]. The Rolls-Royce finite element solver SC03 is employed for solid domain thermal analysis. The code is specifically tailored for turbo-machinery applications, it provides a wide range of specialist thermal modeling capabilities [18,19]. 


\section{Fluid Domain}

All models of the internal air system cavities are constructed in a form of $3 \mathrm{D}$ periodical axisymmetric $0.4^{\circ}$ sectors. Consequently, the obtained results can be regarded as 2D axisymmetric. Such 3D geometrical features as bolts are not included, but cooling holes and nozzles are accounted for in a form of axisymmetric slots with the same opening areas. Exhaustive mesh dependency studies have not been carried with all possible operating conditions encountered during the coupled calculations. However, the mesh density in all the cavities is consistent with the previous studies of the pre-swirl cavity [20]. The rotational Reynolds numbers corresponding to the pre-swirl nozzle radius and inlet conditions for high, intermediate and low power conditions are $4.0244 \cdot 10^{7}, 2.3644 \cdot 10^{7}$ and $0.7375 \cdot 10^{7}$. In all fluid domains we apply the $k-\varepsilon$ turbulence model with wall functions. The choice of turbulence model is based on the previous heat transfer studies for the pre-swirl system reported in [20]. In those studies, acceptable results were obtained compared to pre-swirl test rig measurements. Near wall regions are approximated with the standard wall functions due to time limitations of a coupled fluid-structure thermal transient analysis. The wall mesh $y+$ values are kept in the acceptable range between 30 and 150 over almost the entire set of the coupled walls and models, as depicted in Fig. 2. Given the complexity of the geometries and the time invested in generating these meshes, the $y+$ values are considered to be satisfactory. The boundary conditions

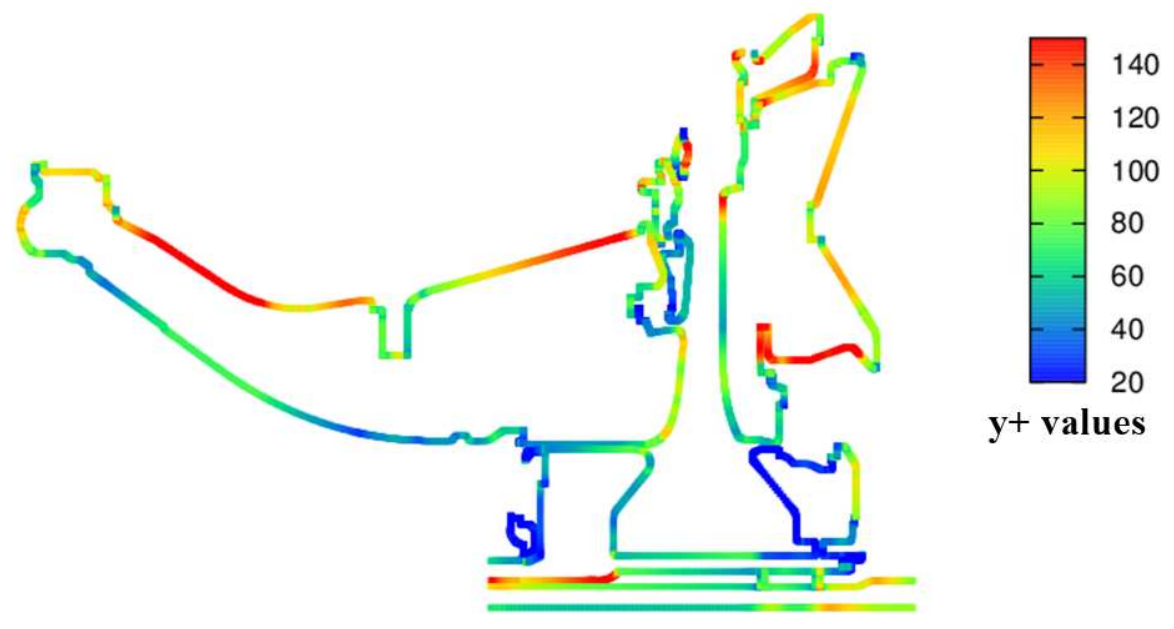

Fig. 2. y+ values at maximum power condition.

are based on our best understanding of the secondary air system flow environment, consistent with the engine test data. Inlet temperatures evolve during the coupled calculations, but the mass flow rates and the pressures are predicted. In all the fluid models we also specify either the flow angle or the swirl velocity at inlets. Static pressure is prescribed at a single outlet in each of the domains, mass flow outlet is specified at the remaining outlets. Detailed flow structure, shown as the stream function for the high power condition of each of the fluid domains, is given in Fig. 3.

Most prominent flow features are captured in all of the fluid domains. Similar flow structures are observed at low and intermediate power operating conditions. Note also that the inlet and outlet boundaries are marked with blue and red colored arrows respectively. All CFD simulations are carried out to second order accuracy of discretization using the HYDRA code. HYDRA is an in-house unstructured finite volume code for compressible Reynolds averaged Navier-Stokes equations [21]. A density based non-linear steady state solver with block Jacobi preconditioning and five stage Runge-Kutta scheme is employed throughout simulation. Convergence is accelerated with an edge collapsing based multi-grid smoothing. Oplus (Oxford parallel libraries for unstructured solvers) library, a part of HYDRA suite of codes, is employed for parallel execution. 


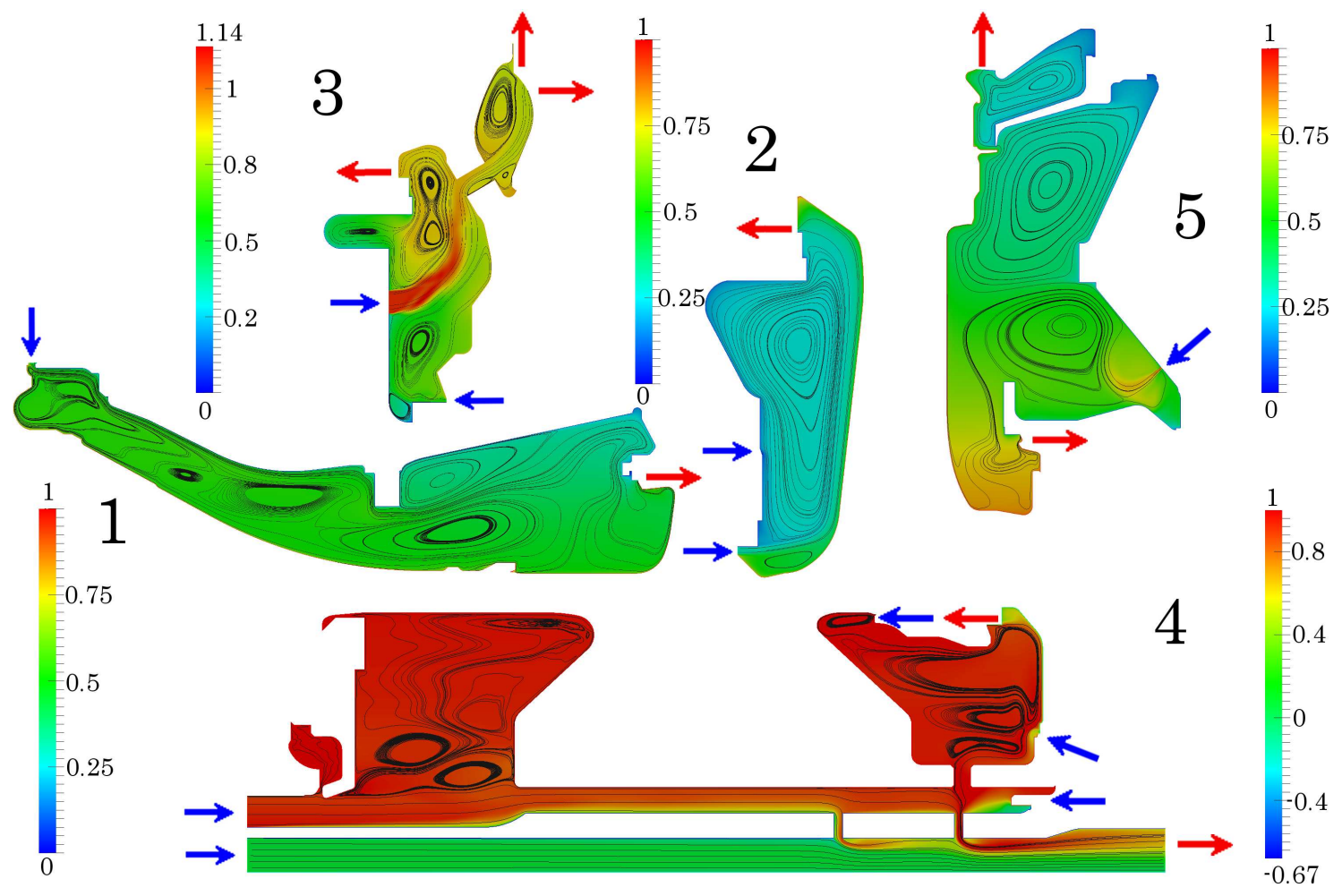

Fig. 3. Stream lines and swirl ratio at maximum power condition in drive cone (1), pre-swirl lower (2), pre-swirl upper (3), bore (4) and rear (5) cavities. Inlet and outlet boundaries are marked by blue and red arrows.

\section{Transient Cycle Definition}

Thermal transient analysis is carried out through an engine flight cycle, which is defined in terms of three shaft speeds. The cycle definition for the HP turbine rotation speed $\Omega_{N H}$ is shown in Fig. 4. The engine test cycle consists of two pairs

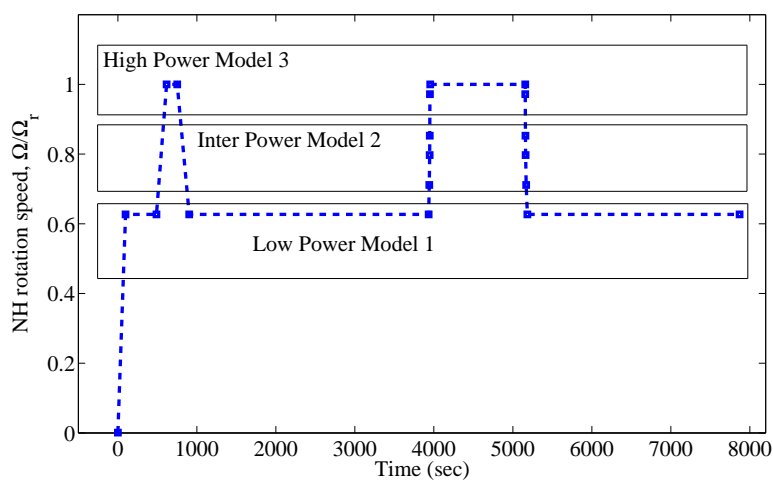

Fig. 4. Transient cycle definition for HP rotor disk. 
Table 1. Grid sizes of fluid domains at three operating conditions.

\begin{tabular}{cccccc}
\hline Condition & $(1)$ & $(2)$ & $(3)$ & $(4)$ & $(5)$ \\
\hline Low & 266,584 & 34,876 & 104,720 & 107,328 & 196,764 \\
& & & & & \\
Int & 266,584 & 40,516 & 110,720 & 107,328 & 214,644 \\
& & & & & \\
High & 275,912 & 65,848 & 120,456 & 135,720 & 235,164 \\
\hline
\end{tabular}

of acceleration and deceleration periods, the first is shorter than the second. The cycle starts with a ramp from stand-still state moving towards the idle condition, then accelerated to the maximum take-off, and finally decelerated to the low power condition. This sequence is repeated for the second pair of acceleration and deceleration periods. For clarity, a constant rotation speed condition is referred to as a "steady ramp", whereas a transient rotation speed is referred to as an "unsteady ramp". The low and maximum power conditions are illustrated in Fig. 4. The whole cycle is subdivided into 19 ramp points. For all of these ramp points we specify operational environmental parameters as a function of the rotation speeds, such as mass flow rates, pressures and temperatures. A linear variation of the operational boundary conditions is assumed between the ramp points. Significant changes in mass flow rates and pressures at the inlet and outlet boundaries are also accounted for by creating distinct CFD models. Thus, three CFD models are created for each cavity. Different grids at each operating condition reflect the need to keep near-wall mesh spacings of the size within the range recommended for standard wall functions while maintaining a proper level of grid quality and resolution. Grid size of the models is provided in Tab. 1. Note that the solid mesh portion amounts to less than $2 \%$ of the overall coupled domain size.

Each CFD model's inlet and outlet mass flow rates correspond to low, maximum and intermediate power conditions, as indicated in Fig. 4. Linear interpolation in time using predictions of two models is carried out in the transition zones between power conditions. In order to reduce the simulation time we employ only steady-state fluid domain solutions, which is a widely accepted industrial practice. This simplification, referred to as quasi-steady-state approximation, is justified by the large differences between the characteristic time scales of the fluid domain convection and the solid domain conduction processes. Inlet and outlet flow rates are fixed in all CFD solutions but the inlet temperatures vary to match the upstream one-dimensional flow network exit condition. The fluid domain outlet boundaries are also connected to the downstream standard convective correlations supplying total temperature and swirl velocity. During the transient cycle metal boundaries and seal clearances may vary significantly under the thermo-mechanical excitation. The variation can be included in the coupled analysis, see [9], but in this work we opt not to. Instead, the effect of varying seal clearances is accounted for by varying the mass flow rates through the seals in fluid domain calculations, which were predicted by FE model and one dimensional flow network calculations consistent with the engine test data.

\section{SIMULATION RESULTS}

\section{Temperature Distribution}

A typical temperature evolution at the reference point 1 predicted by the coupled simulation is compared to the engine test data in Fig. 6. The reference points of the FE model corresponding to the location of the thermo-couples during the engine test are shown in Fig. 5. To better quantify the observable discrepancies between the predicted and the engine test temperatures, the results are also shown as the temperature difference between calculated and measured data. The differences at three points near to blade root 2, inside the the rear cavity 4 and the pre-swirl inner cavity 3 are shown in Figs. 7, 8 and 9 correspondingly. Zero difference in these plots corresponds to a good agreement between the predicted and measured engine test data. For the point near the blade root (Fig. 7), the results of coupled simulation match those of uncoupled. However, 


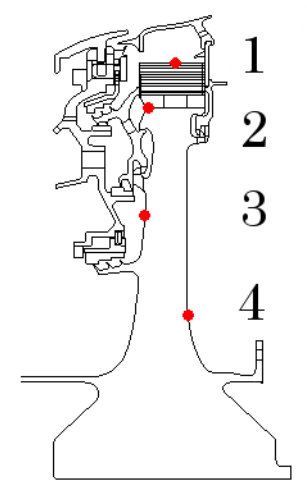

Fig. 5. Location of the reference points on high pressure turbine disk.

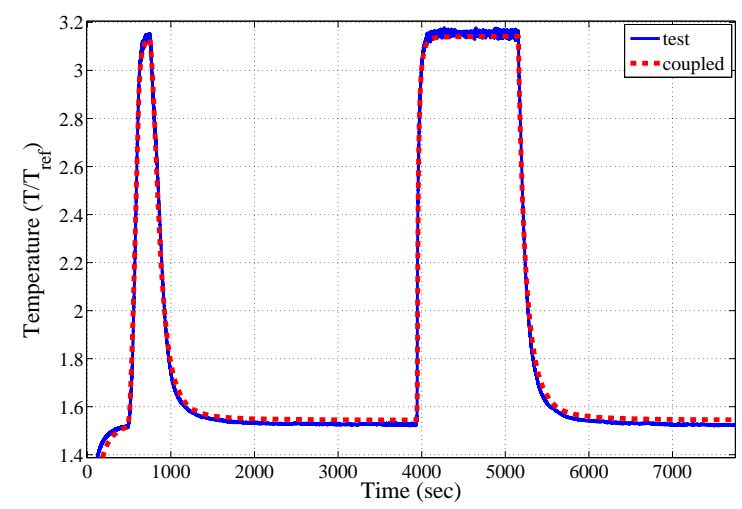

Fig. 6. Temperature evolution at reference point 1 . The coupled prediction is compared to the engine test measurement.

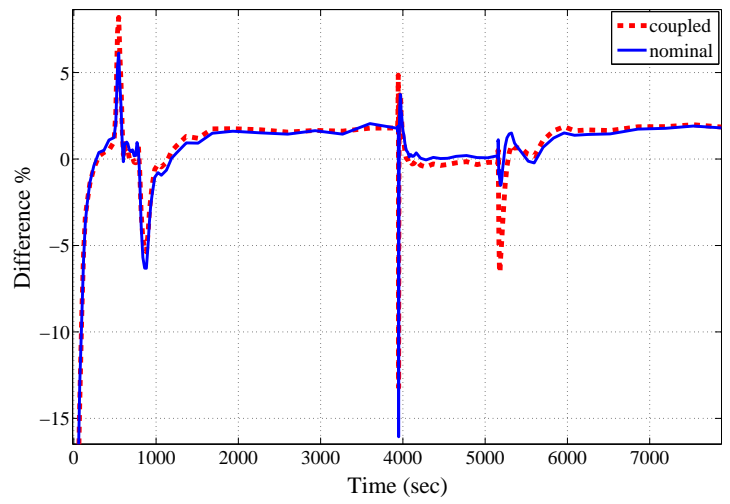

Fig. 7. Nominal and coupled prediction differences with the measured temperature next to the blade root shown as a percentage of the test data. 


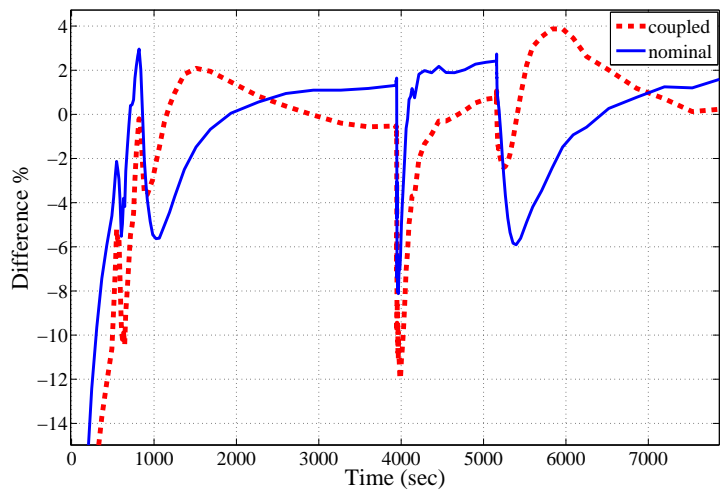

Fig. 8. Nominal and coupled prediction differences with the measured temperature inside the rear cavity at inner radius shown as a percent-

age of the test data.

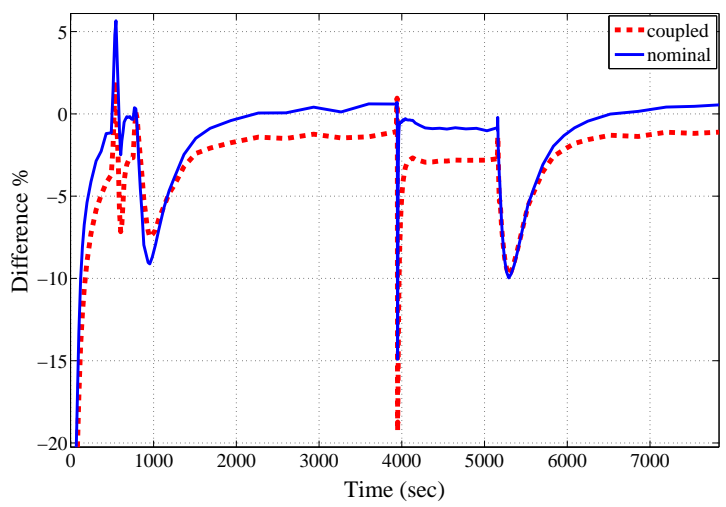

Fig. 9. Nominal and coupled prediction differences with the measured temperature inside the pre-swirl inner cavity shown as a percentage

of the test data.

at other two points (Fig. 8 and Fig. 9), the coupled results temperature is under-predicted. The fast transient response has not been investigated in detail in this study. Since the baseline model uses more ramp points than the coupled one in the fast acceleration and decelerations, the differences in time response between the reference and coupled models are expected. Some of the discrepancies with the test data could be explained by the fact that we neglect some of the 3D features by making axisymmetric assumption for our fluid domain. The misrepresented $3 D$ features add significant windage heating to the fluid domain. In the baseline model they are accounted for by model matching factors in the heat transfer correlations, hence the results better match the engine test data, specifically at the location inside the pre-swirl inner cavity (Fig. 9). Thus, a better agreement with the test data might be obtained with 3D CFD calculation at a price of significantly longer computation time.

The differences between the baseline and coupled predictions are also depicted in the contour plots of Figs. 10 and 11 . The instantaneous temperature contour plots for the baseline model and the coupled solution are compared at $t=4700 \mathrm{sec}$, which corresponds to the maximum power ramp. The contour plot range is normalized with HP first compressor inlet, $T 26$ and HP last stage outlet, T30 temperatures. Similar observations can be made looking at the two contour plots. The coupled calculation temperature profiles are close to the baseline model predictions except for the areas near the drive cone cavity, marked with red boxes in Fig. 11. The coupled calculations show lower temperatures than the baseline model mainly due to 


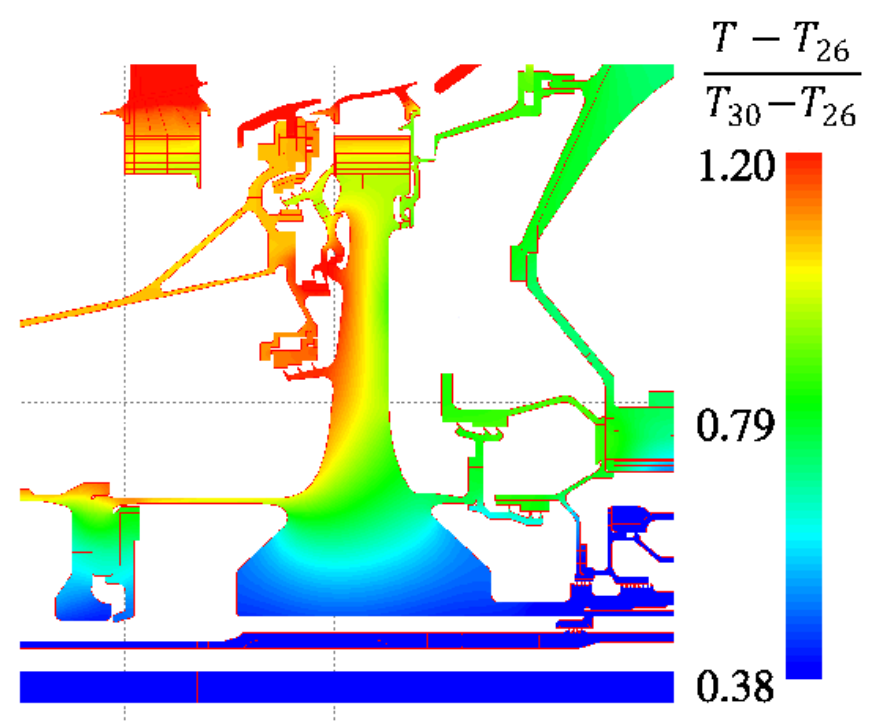

Fig. 10. Metal temperature profile predicted at maximum power condition $t=4700 \mathrm{sec}$ by the baseline model.

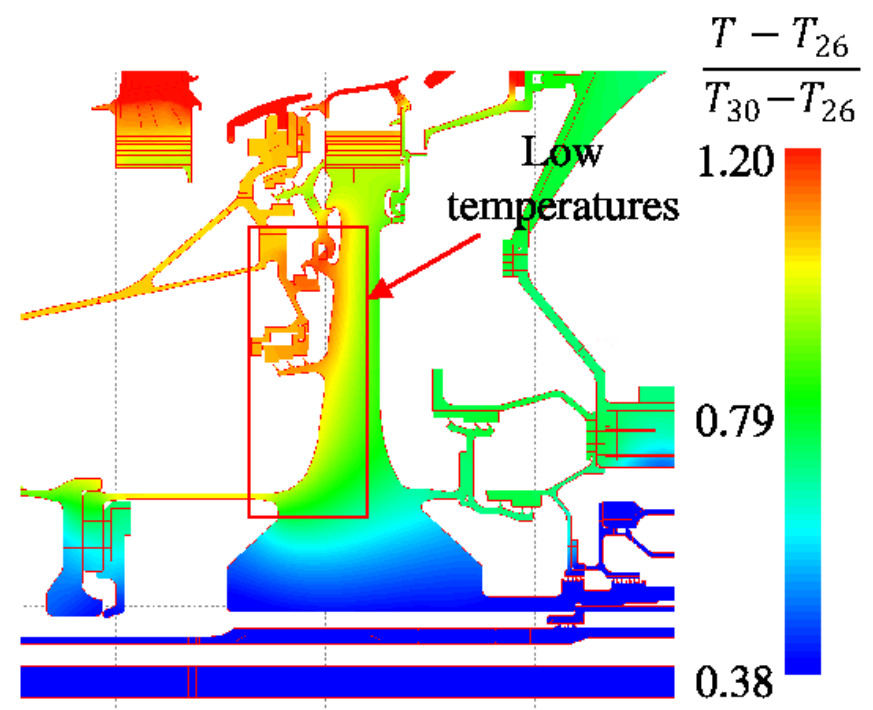

Fig. 11. Metal temperature profile predicted at maximum power condition $t=4700 \mathrm{sec}$ by the coupled method.

the fact we neglect some of the non-axisymmetric geometric features. However, regardless of the axisymmetric limitations, the overall temperature predictions of the coupled approach is as good as the uncoupled baseline calculations, especially in the steady ramps regions. This does not hold true for the unsteady ramp regions, where the predicted temperature levels are still away from the test data. The differences may come from the fluid domain modeling assumptions, the linear interpolation between ramp points, transient behavior of seal clearances and the quasi-steady state approximation. 
Table 2. Performance comparison for two methods.

\section{Method Fluid-Solid Cycles Fluid Solver Calls}

Quasi-Newton $\quad 1392$

Anderson

617

791

\section{Computational Performance}

In this subsection we compare the numerical efficiency of two coupling techniques described in the above. Both experiments are run to the same level of accuracy producing nearly identical temperature histories. A time step is accepted whenever the interface temperature difference becomes smaller than $2.5 \mathrm{~K}$. The Anderson mixing parameters are set to 0.5 for the drive cone cavity (1), 0.4 for two pre-swirl cavities (2)-(3) and 0.3 for both bore and rear cavities (4)-(5). The simulations are conducted on a Linux cluster utilizing in most situations 144 processors.

The wall clock time for the first experiment is approximately 15 days, while its counterpart has completed in 7 to 8 days. The wall clock time is not an accurate performance indicator since it includes the periods of time when the submitted CFD jobs wait in queue. The results of two simulations showing the total number of coupling iterations and fluid solver calls required by each algorithm to satisfy the stopping criterion with the selected tolerance level are given in Tab. 2 . Since separate cavities are executed in parallel, the first number refers to the fluid solver invocation counts that must be carried out sequentially, whereas the second one reflects the computational effort in terms of the total number of fluid solver invocations. The latter includes some extra fluid solver calls needed to estimate the heat transfer coefficient for the quasi-Newton method as well as the calls to two fluid models when interpolation between two ramp points of the transient cycle is required. Thus, provided that the time spent to solve the structural equations is rather negligible compared to the time needed to evaluate heat fluxes, the total number of fluid-solid cycles multiplied by an average cpu time of the largest or the slowest to converge cavity provides a rough estimate of the total duration of coupled simulation. The cumulative number of iterations and fluid solver calls relative to the time step size are shown in Figs. 12 and 13. The results illustrate a clear algorithmic advantage

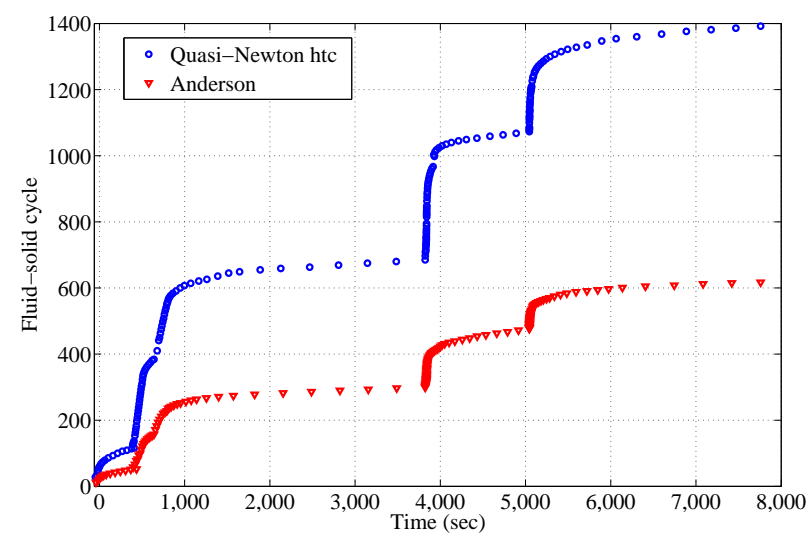

Fig. 12. Cumulative number of fluid-solid cycles.

of the Anderson mixing method over the more established approach formulated on the estimates of heat transfer coefficient. 


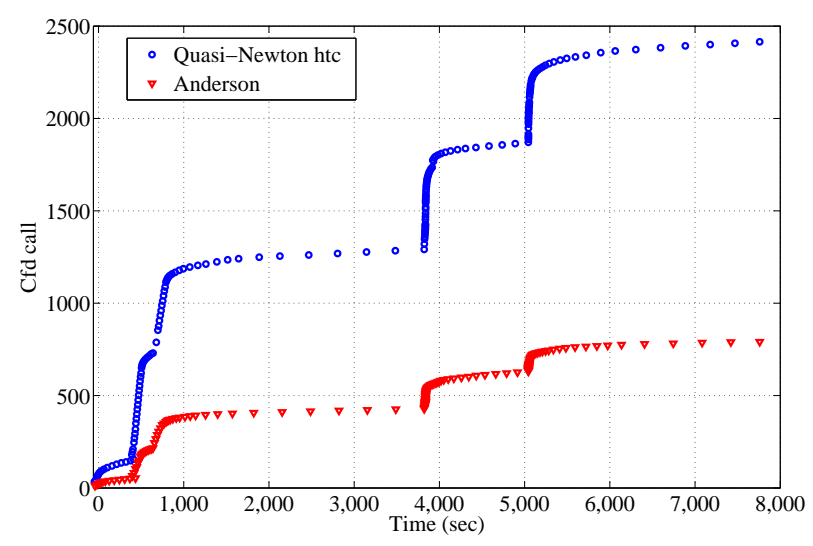

Fig. 13. Total number fluid domain calls.

Our current FORTRAN implementation of the method written as an interface solver between SC03 and HYDRA codes is able to reproduce the performance benefits observed in [17] on a large industrial scale model coupled to five cavities.

\section{CONCLUSION}

In this paper we present the transient aero-thermal analysis of a gas turbine internal air system through an engine flight cycle that features multiple interconnected fluid domains surrounding HP turbine disk with the adjacent structures. Strongly coupled fluid-structure thermal problems require significant computational effort to resolve all nonlinearities on the interface. Simulations grow more computationally intensive as multiple CFD domains are included in the analysis. A new coupling methodology for transient conjugate heat transfer analysis, inspired by the Anderson mixing method, is demonstrated on the selected test case. The new method extracts higher quality and more recent information on heat transfer coefficients directly from the nonlinear iteration sequence. History of iterations from previous time steps is efficiently reused to improve the convergence rate. In addition, formulation of the problem on the interface offers a correct treatment of the nonlinear interactions between multiple interconnected fluid domains, including both coupling through the solid domain walls and the inlet-outlet interdependencies. Overall, this new solution approach compares well to the present technique based on the convective heat flux formulation. The results show that the frequency of exchanges between fluid and solid solvers is considerably reduced leading to a $\mathrm{x} 2$ speedup in overall simulation time. This research is a further step towards the virtual engine modeling, which will eventually eliminate the need for many user-specified correlations-based boundary conditions. Further validation on industrial test problems is still needed as well as an extension of the current work to include the effects of the solid domain deflections and more accurate 3D geometries.

\section{Achnowledgements}

The authors would like to acknowledge Rolls-Royce plc with Engineering and Physical Sciences Research Council for providing financial and technical support for this work.

\section{References}

[1] Chew, J., and Hills, N., 2007. "Computational fluid dynamics for turbomachinery internal air systems". Phylosophical Transactions of the Royal Society, 365, pp. 2587-2611. 
[2] Chew, J., and Hills, N., 2009. "Computational fluid dynamics and virtual aeroengine modelling". Proceedings of the Institution of Mechanical Engineers, Part C: Journal of Mechanical Engineering Science, 223(12), pp. 2821-2834.

[3] Montenay, A., Paté, L., and Deboué, J., 2000. "Conjugate heat transfer analysis of an engine internal cavity". ASME Paper No. 2000-GT-282.

[4] Verdicchio, J., Chew, J., and Hills, N., 2001. "Coupled fluid/solid heat transfer computation for turbine discs". ASME Paper No. 2001-GT-0205.

[5] Okita, Y., and Yamawaki, S., 2002. "Conjugate heat transfer analysis of turbine rotor-stator systems". ASME Paper No. 2002-GT-30615.

[6] Mirzamoghadam, A., and Xiao, Z., 2002. "Flow and heat transfer in an industrial rotor-stator rim sealing cavity". Journal of Engineering for Gas Turbines and Power, 124(1), pp. 125-132.

[7] Illingworth, J., Hills, N., and Barnes, C., 2005. "3D fluid-solid heat transfer coupling of an aero-engine preswirl system". ASME Paper No. 2005-GT-68939.

[8] Sun, Z., Chew, J., Hills, N., Volkov, K., and Barnes, C., 2010. "Efficient finite element analysis/computational fluid dynamics coupling for engineering applications". Journal of Turbomachinery, 132, pp. 031016-1-031016-9.

[9] Amirante, D., and Hills, N., 2010. "Thermo-mechanical FEA/CFD coupling of an interstage seal cavity using torsional spring analogy". ASME Paper No. 2010-GT-22684.

[10] Anderson, D., 1965. "Iterative procedures for nonlinear integral equations". Journal of the Association of Computing Machinery, 12, pp. 547-560.

[11] Patankar, S., 1980. Numerical Heat Transfer and Fluid Flow. McGraw Hill, New York NY.

[12] Bohn, D., Heurer, T., and Kusterer, K., 2003. "Conjugate flow and heat transfer investigation of a turbo-charger - Part I: Numerical results". ASME Paper No. 2003-GT-38445.

[13] Giles, M., 1997. "Stability analysis of numerical interface conditions in fluid-structure thermal analysis". International Journal for Numerical Methods in Fluids, 25, pp. 421-436.

[14] Roe, B., Haselbacher, A., and Geubelle, P., 2007. "Stability of fluid-structure thermal simulations on moving grids". International Journal for Numerical Methods in Fluids, 54, pp. 1097-1117.

[15] Lassaux, G., Daux, S., and Descamps, L., 2004. "Conjugate heat transfer analysis of a tri-dimensional turbine blade internal cavity". 24th ICAS.

[16] Verstraete, T., Alsalihi, Z., and van den Braembussche, R., 2007. "Numerical study of the heat transfer in micro gas turbines". Journal of Turbomachinery, 29, pp. 835-841.

[17] Ganine, V., Hills, N., and Lapworth, B., 2012. "Nonlinear acceleration of coupled fluid-structure transient thermal problems by Anderson mixing". International Journal for Numerical Methods in Fluids.

[18] Dixon, J., Verdicchio, J., Benito, D., Karl, A., and Tham, K., 2004. "Recent developments in gas turbine component temperature prediction methods, using computational fluid dynamics and optimization tools, in conjunction with more conventional finite element analysis techniques". In Proceedings of the Institution of Mechanical Engineers, Journal of Power and Energy, Vol. 128(4).

[19] Armstrong, I., and Edmunds, T., 1989. "Fully automatic analysis in the industrial environment". International Conference on Quality Assurance and Standards, NAFEMS.

[20] Javiya, U., Chew, J., Hills, N., Zhou, L., Wilson, M., and Lock, G., 2010. "CFD analysis of flow and heat transfer in a direct transfer pre-swirl system". ASME Paper No. 20102-GT-22964.

[21] Moinier, P., 1999. "Algorithm development for an unstructured viscous flow solver". PhD thesis, University of Oxford. 